\title{
Establishment and characterization of patient-derived primary cell lines as preclinical models for gallbladder carcinoma
}

\author{
Feiling Feng" ${ }^{1 \#}$, Chuncui Huang ${ }^{2 \#}$, Mingjia Xiao ${ }^{1 \#}$, Huizhen Wang ${ }^{3 \#}$, Qingxiang Gao ${ }^{1}$, Zishuo Chen ${ }^{3}$, \\ Xiaoya $\mathrm{Xu}^{3}$, Jun Zhou ${ }^{3}$, Fugen $\mathrm{Li}^{3}$, Yan $\mathrm{Li}^{4}$, Dadong Zhang ${ }^{3}$, Yanxin Chang ${ }^{5}$, Xiaoqing Jiang ${ }^{1}$ \\ ${ }^{1}$ Department of Biliary I, Shanghai Eastern Hepatobiliary Surgery Hospital, Navy Military Medical University, Shanghai 200438, China; ${ }^{2}$ Institute of \\ Biophysics, Chinese Academy of Sciences, Beijing 100101, China; ${ }^{3}$ Research and Development Institute of Precision Medicine, 3D Medicines Inc., \\ Shanghai 201114, China; ${ }^{4}$ University of Chinese Academy of Sciences, Beijing 100049, China; ${ }^{5}$ Biliary Tract Surgery Department, Shanghai Eastern \\ Hepatobiliary Surgery Hospital, Navy Military Medical University, Shanghai 200438, China \\ Contributions: (I) Conception and design: D Zhang, Y Chang, X Jiang; (II) Administrative support: F Li, Y Li; (III) Provision of study materials or \\ patients: F Feng, M Xiao, Q Gao; (IV) Collection and assembly of data: Z Chen, X Xu, J Zhou; (V) Data analysis and interpretation: F Feng, C \\ Huang, M Xiao, H Wang; (VI) Manuscript writing: All authors; (VII) Final approval of manuscript: All authors. \\ \#These authors contributed equally to this work. \\ Correspondence to: Professor Xiaoqing Jiang, MD. Department of Biliary I, Shanghai Eastern Hepatobiliary Surgery Hospital, Navy Military Medical \\ University, 225 Changhai Road, Shanghai 200438, China. Email: xqjiang_dandaoyike@163.com; Yanxin Chang, MD. Biliary Tract Surgery Department, \\ Shanghai Eastern Hepatobiliary Surgery Hospital, Navy Military Medical University, Shanghai 200438, China. Email: pycmu402@163.com; \\ Dadong Zhang, PhD. Research and Development Institute of Precision Medicine, 3D Medicines Inc., Room 201, Building 2A, No. 158 Xin Junhuan \\ Road, Pujiang Hi-tech Park, Shanghai 201114, China. Email: dadong.zhang@3dmedcare.com.
}

Background: Gallbladder carcinoma (GBC) is one of the most lethal malignancies which do not have a targeted drug in the clinic. Patient-derived primary cell lines (PDCs) are useful in assessment of cancer complexity and heterogeneity, drug-sensitivity tests, and personalized-drug-selection guidance. The aim of this study is to establish GBC PDCs and characterize their biological features.

Methods: The characterization of PDCs was defined by morphology, growth kinetics, chromosomal analysis, short tandem repeat (STR) analysis, RNA-seq and tumorigenicity. Glycosylation of PDCs derived from GBC was first studied, and the PDC model's performance were also tested and evaluated using seven molecular target inhibitors.

Results: Three novel GBC cell lines from three GBC patients were successfully established and denoted as JXQ-3D-902R4, JXQ-3D-4494R, and JXQ-3D-4786R. These cell lines demonstrated the heterogeneous characteristics of tumor morphology and phenotypes which are consistent with primary GBC, such as irregular cell shape, varied chromosomal numbers, and different STR patterns. Moreover, the growth activity and tumorigenicity ability varied among the cell lines, of which JXQ-3D-4494R exhibited the best growth rate. Furthermore, glycan profiling of whole proteins were detected and characterized. Unique $\mathrm{N}$-glycans of each PDC were identified, JXQ-3D-902R4, JXQ-3D-4494R and JXQ-3D-4786R contained ten, four and seven unique glycans, respectively. The epithelial origins of three PDCs were confirmed using RNAseq based on the highly expressed typical epithelial marker genes. Moreover, the drug-sensitivity results demonstrated that the three PDCs exhibited different responses to the seven-most commonly used targeted medicines belonging to three groups: cell-cycle inhibitors, PI3K/AKT/mTOR signaling-pathway inhibitors, and ErbB inhibitors. JXQ-3D-4494R was sensitive to most of the inhibitors, JXQ-3D-4786R was sensitive to ErbB inhibitors, and JXQ-3D-902R4 was sensitive to PI3K/AKT/mTOR inhibitors.

Conclusions: These results indicate that PDCs may be efficient preclinical models for further investigation of the biological behaviors and potential targeted therapies of human GBC.

Keywords: Gallbladder carcinoma (GBC); patient-derived primary cell line (PDC); glycosylation; preclinical model; drug sensitivity 
Submitted Aug 29, 2019. Accepted for publication Jan 18, 2020.

doi: 10.21037/tcr.2020.02.04

View this article at: http://dx.doi.org/10.21037/tcr.2020.02.04

\section{Introduction}

Gallbladder carcinoma (GBC), the most common primary biliary tract cancer (BTC), is one of the most lethal malignancies, which is generated from the gallbladder or cystic duct. GBC accounts for nearly two-thirds of BTCs and is the sixthmost common gastrointestinal tract cancer (1), and is characterized by its aggressive growth and high lethality. In China, GBC is the fifth-ranked cancer of the gastrointestinal tract. The age of high incidence is 50-75 years old, and its incidence in females is about twofold higher than that of males $(2,3)$. The onset of GBC is insidious, difficult to diagnose early, exhibits a high degree of malignancy, and is prone to early metastasis. Consequently, GBC generally carries an extremely poor prognosis because the majority of patients are at an advanced stage at the initial diagnosis. The 5 -year survival rate of GBC is between 5\% and $10 \%$ at advanced stages (4).

Currently, surgical resection in combination with neoadjuvant or adjuvant therapies is still the only potentially curative modality for resectable GBC patients to achieve long-term survival (5). However, GBC resection rate was $69.7 \%$, and the rate of curative resection was $37.7 \%$ from 1998 to 2004 in Japan (6). Unfortunately, only a small proportion of GBC patients are eligible to receive surgical intervention due to organ or liver invasion starting from a relatively early stage (7). A standard treatment for GBC has not yet been established $(8,9)$. Although advances in many therapeutic strategies against GBC have been developed, the prognosis for the majority of patients with GBC has not improved appreciably (9). Therefore, it is important to investigate the specific biological mechanisms underlying GBC and to evaluate the sensitivity of potential drugs to treat GBC.

Recently, patient-derived primary cell lines (PDCs) have been developed and characterized as tumor models for cancer research (10). PDCs, which are isolated directly from resected tumor tissues, have a finite lifespan and limited expansion capacity, maintaining many of the important markers and functions seen in original tissues $(11,12)$. PDC models retain the principal histologic and genetic characteristics of donor tumors and remain stable throughout passages, and are also used to predict clinical outcomes used for preclinical drug evaluation, biomarker identification, biological studies, and personalizedmedicine strategies (13-15). Thus, PDC models are useful for assessment of the complexity and heterogeneity of GBCs, test-drug sensitivities, and for guiding personalizeddrug selection for each patient. At present, there are few established GBC cell lines derived from primary tumors (16), and no studies have characterized glycosylation in these GBC cell lines.

Glycosylation is one of the most common posttranslational protein modifications and is associated with several pathophysiological processes. Aberrant glycosylation may lead to the development of cancer. It have been reported that fucosylation of prostate-specific antigen (PSA) can be used to differentiate aggressive and non-aggressive prostate cancer (17). Moreover, changes in glycan patterns may contribute to identifying stages of colorectal cancer (CRC). The carcinoembryonic-antigen (CEA) glycan levels of $\mathrm{N}$-acetylgalactosamine, mannose, and galactose during stage-II CRC have a significant downward trend compared with those at other stages. Thus, these altered glycans may be indicative of the progression of tumor development (18). Furthermore, N-glycan profiling may help in the determination of the origin of a carcinoma and may help to differentiate normal cells from cancer cells. It has been reported that high-mannose-type and corefucosylated $\mathrm{N}$-glycans are higher in bladder cells than in normal cells (19). The study of various CRC cell lines has also demonstrated that bisecting $\mathrm{N}$-acetylglucosamine is expressed at a high level in the CRC cell line, LIM1215 (20). Thus, these glycans may serve as molecular markers to differentiate different stages of cancer.

Our previous study has demonstrated that three PDCs, derived from the same patient with gallbladder sarcomatoid carcinoma, could be applied to elucidate the sensitivity of cells to targeted agents (21). In this study, we further established three PDCs using fresh primary tumor cells from three surgically resected patients with GBC. Here, we characterized three new human GBC cell lines, including their glycosylation features, and also examined their responses to the seven-most commonly used targeted therapeutic agents. The three novel PDCs could be an 
ideal tool for further investigation of the tumor biology and potential treatment of GBC.

\section{Methods}

\section{PDC establishment and cell culture}

The entire process of PDC establishment was sterile. Fresh gallbladder adenocarcinoma tissues from three patients were washed by cold sterile phosphate buffered saline (PBS) to remove excess blood and connective tissue. Then the tissues were cut into $1 \mathrm{~mm}^{3}$ pieces and washed by cold sterile PBS several times. All pieces were placed in $10 \mathrm{~cm}^{2}$ dishes and incubated in DMEM/F12 complete growth medium which consisted of 90\% DMEM/F12 $\left(\right.$ Gibco $^{\mathrm{TM}}$, C11330500BT) with 10\% fetal bovine serum (FBS) (Gibco; Thermo Fisher Scientific, Inc., Waltham, MA, USA), and with $1 \%$ non-essential amino acids (Gibco $\left.{ }^{\mathrm{TM}}, 11140076\right)$ and $1 \%$ penicillin $(100 \mathrm{U} / \mathrm{mL}) /$ streptomycin $(100 \mu \mathrm{g} / \mathrm{mL})$ (Gibco $^{\mathrm{TM}}$, 15140-122; Thermo Fisher Scientific, Inc.). When the cells reached approximately $30 \%$ confluency, the supernatant and excessive tissues were removed and the cells were detached by $0.25 \%$ trypsin-EDTA $\left(\right.$ Gibco $^{\mathrm{TM}}$, 25200072; Thermo Fisher Scientific, Inc.) and transferred into 24-well plates. After 5-6 passages tumor cells were effectively purified, then the cells were scale-up cultured in DMEM/F12 complete growth medium and were grown in a 377 incubator with $5 \% \mathrm{CO}_{2}$. After establishment, the three PDCs were stored at low passages. The passage of each PDC used in the following experiment was different and the passage information for each PDC in the assays ranged between 15 and 30 .

\section{Cell morphology, karyotypes and short tandem repeat (STR) analysis}

The morphologies of three PDCs (JXQ-3D-4494R, JXQ-3D-4786R and JXQ-3D-902R4) were detected by microscopy. Pictures at 40×, 100x and 200x magnifications were taken after changing media after every passage at an appropriate cell density. Subsequently, karyotype analysis and STR sequence were detected before experiments to ensure that the cells were homogeneous. Three PDCs (JXQ3D-4494R, JXQ-3D-4786R and JXQ-3D-902R4) were trypsinized and plated to new $10 \mathrm{~cm}^{2}$ dishes. The medium were changed with the addition of $0.2 \mu \mathrm{g} / \mathrm{mL}$ colchicine when the cells reached $80-90 \%$ confluence. After $1 \mathrm{~h}$ incubation at $37^{\circ} \mathrm{C}$, the cells were trypsinized, centrifuged and resuspended gently with $7 \mathrm{ml}$ pre-warmed $0.075 \mathrm{~mol} / \mathrm{L}$ $\mathrm{KCl}$ solution and incubated for 20 minutes in a $37{ }^{\circ} \mathrm{C}$ water bath. After 3 minutes pre-fixed by addition of $1 \mathrm{~mL}$ Carnoy's Fluid, the cells were centrifuged at 1,200 rpm for 5 minutes, resuspended gently with $7 \mathrm{~mL}$ fresh Carnoy's Fluid and incubated for 40 minutes in a $37{ }^{\circ} \mathrm{C}$ water bath. Then the cells were centrifuged and resuspended with about $500 \mu \mathrm{L}$ Carnoy's Fluid to an appropriate cell concentrate. Then the suspension were dropped to pre-cooled slices and dried quickly. The slices were stained with $10 \%$ Giemsa for 10 minutes. Pictures of $40 \times$ were taken for at least 20 metaphase cells with a microscope after the slices were dried. Three PDCs (JXQ-3D-4494R, JXQ-3D-4786R and JXQ-3D-902R4) and their origin tissues were taken STR profiling analysis for 20 loci by Shanghai Biowing Applied Biotechnology Co. Ltd.

\section{Colony-formation assay}

Cells were plated at 500 cells per well in six-well plates with complete DMEM/F12 medium and were incubated in a $37^{\circ} \mathrm{C}$ incubator with $5 \% \mathrm{CO}_{2}$ for 10 days. On day 10 , the cells were rinsed with PBS and fixed with methanol for $10 \mathrm{~min}$. Then, the cells were stained with $0.1 \%$ crystal violet for $10 \mathrm{~min}$ and the numbers of colonies were counted.

\section{Cell proliferation assay}

Three PDCs (JXQ-3D-4494R, JXQ-3D-4786R and JXQ3D-902R4) and no-cell controls were plated in six 96-well plates and were biologically replicated across three wells. Three PDCs were cultured in three densities at 4,000, 2,000 and 1,000 cells/well to determine the optimal plating density. The experimental design is shown in Figure S1. For plates that required incubation times that were longer than $48 \mathrm{~h}$, the medium was replaced every $48 \mathrm{~h}$. The entire incubation process was maintained under sterile conditions during addition of reagents and incubations to avoid microbial contaminants. The number of viable cells from each plate was determined at 24, 48, 72, 96, 120 and $144 \mathrm{~h}$ by alarma blue (kept from direct light) and the fluorescence was determined at 530-560 nm excitation wavelength and $590 \mathrm{~nm}$ emission wavelength by a microplate reader after a $90 \mathrm{~min}$ incubation at $37{ }^{\circ} \mathrm{C}$. Then, Graphpad Prism5 software was used to calculate the population-doubling times of each cell line at different cell densities. With 
the assumptions that all cells grew exponentially and that growth consisted of a constant doubling time, nonlinear regression was chosen to analyze population-doubling times.

\section{RNA-sequencing}

RNA-seq was performed as previously described (22). Randomly interrupted mRNA and the first strand cDNA were synthesized, and then a second cDNA strand was synthesized. RNA-seq library construction, hybrid capture and sequencing were performed at WuXi Next CODE (Shanghai, China). RNA-seq was performed using an Illumina HiSeq X Ten PE150 instrument.

\section{$N$-glycosylation modification of proteins assay}

Whole proteins from cells were extracted with densitygradient centrifugation and differential centrifugation technology. After harvesting and washing $1 \times 10^{7}$ cells in PBS, the cell pellets were resuspended in a homogenization buffer consisting of $0.25 \mathrm{M}$ sucrose, $20 \mathrm{mM}$ HEPES$\mathrm{KOH}$, and $\mathrm{pH} 7.4$ with a protein-inhibitor cocktail before freezing and thawing the samples. Cell lysates were centrifuged at 2,000 $\mathrm{g}$ for $10 \mathrm{~min}$ to remove insoluble cell debris before ultra-centrifugation at 200,000 $\mathrm{g}$ for $45 \mathrm{~min}$ at $4^{\circ} \mathrm{C}$ to collect the cytosolic fractions. Second step, release $\mathrm{N}$-glycans. Glycoproteins were first reduced and carboxymethylated in the presence of dithiothreitol (DTT) and iodoacetamide (IAA) followed by dialysis in 50 $\mathrm{mM}$ Ambic buffer for $24-48 \mathrm{~h}$ at $4^{\circ} \mathrm{C}$. Glycoproteins were then digested by trypsin at $37^{\circ} \mathrm{C}$ overnight. The resulting glycopeptides were applied to a Sep-Pak C18 cartridge which was conditioned successively with $5 \%$ acetic acid and $100 \%$ propan-1-ol, and eluted stepwise with $20 \%$ and $40 \%$ propan-1-ol solution. The propan-1-ol fractions were digested with PNGase F (New England BioLabs, Inc., Ipswich, MA, USA) at $37^{\circ} \mathrm{C}$ for $20-24 \mathrm{~h}$. Separation of the $\mathrm{N}$-glycans from the digestion mixture was made by using the C18 Sep-Pak and the solvent system propan-1-ol/5\% acetic acid. Last, multistage matrix-assisted laser desorption/ ionization time of flight mass spectrometry (MALDI-TOFMS) analysis (23). Lyophilized derivatization $\mathrm{N}$-glycans were resuspended in $10 \mu \mathrm{L}$ methanol. Then a $1 \mu \mathrm{L}$ aliquot and $20 \mathrm{mg} / \mathrm{mL}$ dihydroxybenzoic acid (DHB) were spotted on a stainless-steel $\mu$ Focus MALDI target plate and mixed well. After being air-dried, the obtained $\mathrm{N}$-glycans were characterized by MALDI-TOF-MS.

\section{Tumorigenesis ability of PDCs}

Three PDCs (JXQ-3D-4494R, JXQ-3D-4786R and JXQ3D-902R4) were trypsinized, counted and diluted into $1 \times 10^{7}$ cells $/ 75 \mu \mathrm{L}$ with complete culture media. Then, an equal volume of matrix gel was mixed with the cell suspension on ice to generate a suspension with a density of $1 \times 10^{7}$ cells $/ 150 \mu \mathrm{L}$. The cell suspension was subcutaneously injected into four sites, including both flanks of the scapula and the outer thigh of 4-weeks-ol female Balb/c nude mice (Shanghai Slake Experimental Animal Co., LTD) for $150 \mu \mathrm{L}$ per site. Tumor sizes were measured once a week and calculated by volume $=$ width $^{2} \times$ length $/ 2$. The mice were sacrificed when the tumor reached $\sim 1,000 \mathrm{~mm}^{3}$. All animal experiments and procedures were carried out in accordance with the institutional protocol approved by the Medicine Institutional Animal Care.

\section{In vitro anti-proliferation assay of PDCs}

The three PDCs were further characterized by analyzing their responses to chemo-agents. Seven compounds (ribociclib, palbociclib, MK2206, everolimus, PI-103, lapatinib and dacomitinib) were tested. A vehicle control and positive control were also produced at the same time. The seven compounds were first dissolved in DMSO, and then each compound was diluted into nine doses by gradient dilution at a gradient of $1: 3.16$. Then, the compounds were sequentially diluted by DMEM/F12 complete growth medium to the final concentration 10x. The maximum concentration of the nine doses for the seven compounds was as follows: ribociclib $(100 \mu M)$, palbociclib $(200 \mu M)$, MK2206 (30 $\mu \mathrm{M})$, everolimus $(1 \mu \mathrm{M})$, PI-103 (50 $\mu \mathrm{M})$, lapatinib $(50 \mu M)$ and dacomitinib $(100 \mu M)$. The original cell density of three PDCs were as follows: JXQ-3D-4494R, 1,000 cells/well; JXQ-3D-4786R, 4000 cells/well; and JXQ3D-902R4, 2,000 cells/well. Cell viability was measured using a Cell Titer-Glo Luminescent Cell Viability Assay (Promega, USA) after $144 \mathrm{~h}$ incubation. Additionally, the $50 \%$ inhibition of cell viability (IC50) was calculated from a four-parameter curve analysis and was determined from a 
A GBC PDCs

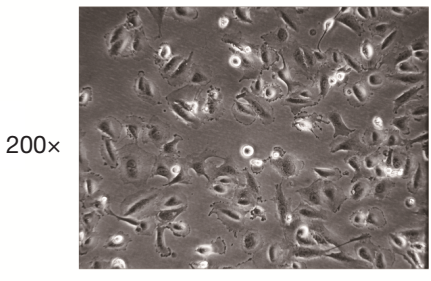

JXQ-3D-902R4

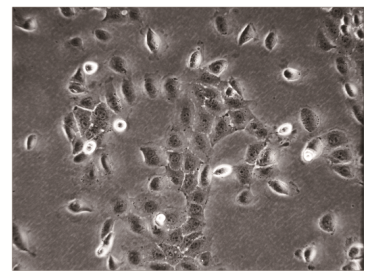

JXQ-3D-4494R

B

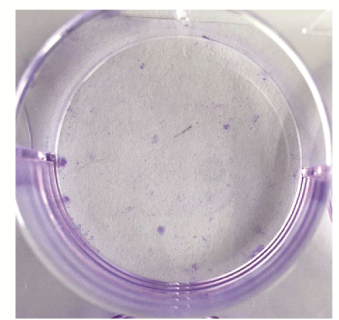

JXQ-3D-902R4

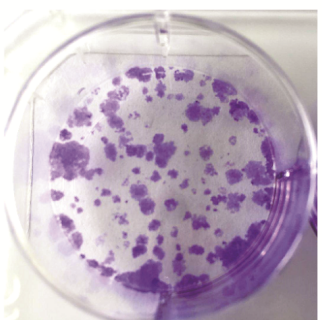

JXQ-3D-4494R

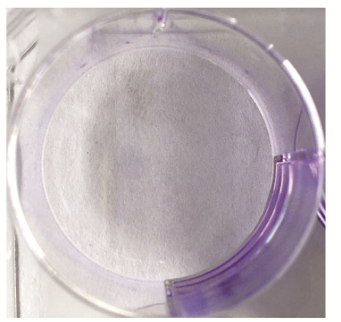

JXQ-3D-4786R

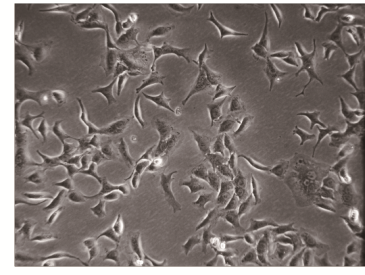

JXQ-3D-4786R

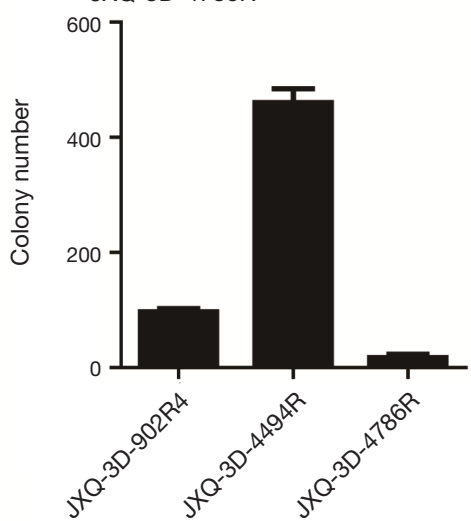

C

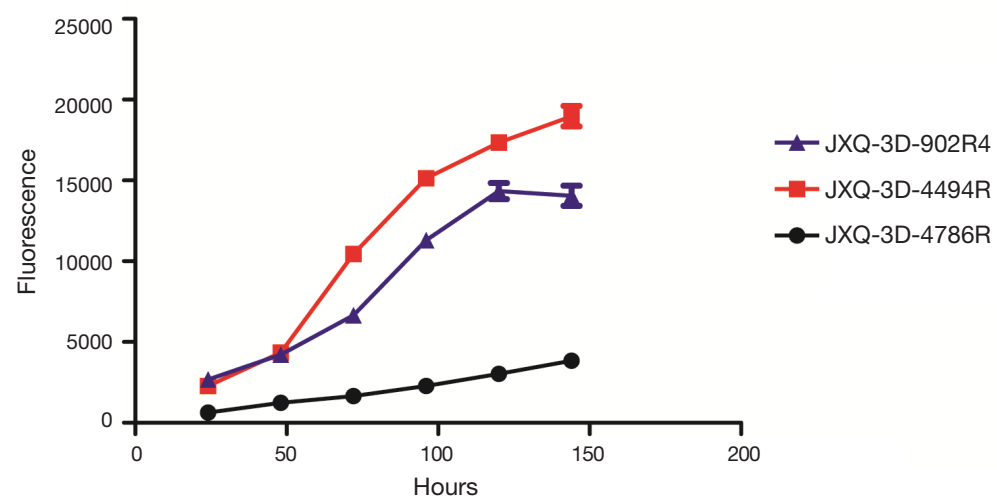

Figure 1 Biological characteristics of three GBC PDCs. The three GBC PDCs (JXQ-3D-902R4, JXQ-3D-4494R, and JXQ-3D-4786R) were derived from three gallbladder adenocarcinoma patients. The cell morphology (A), colony formation activity (B), and proliferation (C) of three GBC PDCs are shown. For colony formation assay (B), a colony is defined as a cluster of approximately 50 cells which can often be determined microscopically. For cell proliferation assay (C), the fluorescence of $y$-axis represented the cell densities of three PDCs at origin cell density of 2,000 cells/well. Magnification, 200x. Error bars represent mean +/- SD from three replicates. GBC, gallbladder carcinoma; PDC, patient-derived primary cell line; SD, standard deviation.

minimum of three experiments.

\section{Results}

\section{Establishment of GBC PDCs}

Three PDCs were successfully established in vitro from human tumor tissues which were obtained from surgically resected specimens of three GBC patients, who were further diagnosed as gallbladder adenocarcinoma by pathologists. The three novel GBC PDCs were denoted as JXQ-3D902R4, JXQ-3D-4786R and JXQ-3D-4494R, which were derived from different sites of the three GBC patients (Figure 1A). The patients did not receive any intervention or treatment before surgery. The clinical characteristics of 
Table 1 Clinical information of three patients with gallbladder adenocarcinoma*

\begin{tabular}{|c|c|c|c|c|c|c|c|c|c|c|c|c|c|c|}
\hline Cell line & $\begin{array}{c}\text { Clinical } \\
\text { pathological } \\
\text { diagnosis }\end{array}$ & Sex & Age & Site & Size & TNM & $\begin{array}{l}\text { Tumor } \\
\text { staging }\end{array}$ & $\begin{array}{c}\text { CEA } \\
(\mathrm{ng} / \mathrm{mL})\end{array}$ & $\begin{array}{l}\text { CA19-9 } \\
(\mathrm{U} / \mathrm{mL})\end{array}$ & $\begin{array}{l}\text { CA125 } \\
(\mathrm{U} / \mathrm{mL})\end{array}$ & $\begin{array}{l}\text { CA72-4 } \\
(\mathrm{U} / \mathrm{mL})\end{array}$ & $\begin{array}{c}\text { AFP } \\
(\mathrm{ng} / \mathrm{mL})\end{array}$ & $\begin{array}{c}\text { Total } \\
\text { bilirubin } \\
(\mu \mathrm{mol} / \mathrm{L})\end{array}$ & $\begin{array}{c}\text { TBA } \\
(\mu \mathrm{mol} / \mathrm{L})\end{array}$ \\
\hline $\begin{array}{l}\text { JXQ-3D- } \\
4494 R\end{array}$ & $\begin{array}{c}\text { Gallbladder } \\
\text { adenocarcinoma }\end{array}$ & Male & 65 & Gallbladder & $5 \mathrm{~cm}^{-}$ & T3N0M1 & $\mathrm{IVb}$ & 1.5 & 7.7 & 18.8 & 2.3 & 2.2 & 6.4 & 5.6 \\
\hline
\end{tabular}

*All three patients were initially diagnosed with gallbladder adenocarcinoma, and they did not receive any intervention or treatment before surgery. TNM, tumor, nodes, metastasis; CEA, carcinoembryonic antigen; CA, carbohydrate antigen; AFP, alpha fetoprotein; TBA, total bile acid.

Table 2 Karyotype analysis of three newly established PDCs

\begin{tabular}{ll}
\hline Cell line & Karyotype information* (number of chromosomes/number of cells) \\
\hline JXQ-3D-902R4 & $70 / 1,71 / 4,72 / 2,73 / 1,74 / 1,75 / 2,76 / 2,78 / 1,80 / 4,81 / 1,83 / 1$ \\
JXQ-3D-4786R & $54 / 1,56 / 1,57 / 1,58 / 2,59 / 1,60 / 2,61 / 1,62 / 3,63 / 2,64 / 2,65 / 1,66 / 1,67 / 1,68 / 1$ \\
JXQ-3D-902R4 & $43 / 1,44 / 1,46 / 1,47 / 2,48 / 3,49 / 4,50 / 3,51 / 4,52 / 1$ \\
\hline
\end{tabular}

*Karyotype analysis of three newly established PDCs was carried out in metaphase in cell division, and 20 cells for each PDC were lysed and the chromosomes were counted. The chromosomes' distribution according the numbers is shown in the table. PDCs, patient-derived primary cell lines.

the GBC patients related to the three PDCs are listed in Table 1 .

\section{Characterization of GBC PDCs}

The morphological characteristics of the three PDCs were observed under phase-contrast microscopy in vitro. All three cultured tumor cells grew in monolayer sheets and adhered strongly to the bottom of dishes. The morphology of three PDCs was a little different. JXQ-3D-902R4 grew in irregular-shaped, partially-fusiform, spindle-shaped single cells varying slightly in size. JXQ-3D-4786R grew mainly in clusters of long polygonal cells that formed branches in many directions, and the morphology of JXQ-3D-4494R was quite different since it exhibited short blunt roundshaped single cells, which were clustered in a way that mimicked pavement confluency in appearance (Figure 1A).

Chromosomal aberrations were observed in all three PDCs. Chromosomal analyses revealed a range of numerical abnormalities with aneuploidy. Most cells of the three PDCs had more than 46 chromosomes except some cells of JXQ-3D-4786R, which had less than 46 chromosomes
(Table 2). Variations in chromosomal number are shown in Table 2. The chromosomal number of JXQ-3D-902R4 ranged between 70 and 83 , with a median of 75 , while the chromosomal number of JXQ-3D-4494R was distributed in the range of 54-68 without an obvious mode, but was mainly distributed between 58 and 64. Finally, JXQ-3D$4786 \mathrm{R}$ had a modal chromosomal number of 49 to 51 with a range of 43-52 (Table 2).

A STR profiling assay of 20 standard STR markers, and Amelogenin gender-determining markers, was performed for the three PDCs (Table 3) and their origin tissues (Table S1). The marker for Amelogenin demonstrated that the JXQ3D-902R4 cell line was from female origin with XX chromosomes, whereas JXQ-3D-4494R and JXQ-3D4786R were of male origin with $\mathrm{XY}$ chromosomes. The identification of new cell lines and the assessment of cross-contamination were carried out through the DSMZ database (https://www.dsmz.de/services/services-humanand-animal-cell-lines/online-str-analysis.html). No cross contamination was found for any cell line. The STR results suggest that the three PDCs are novel GBC cell lines. Comparing the STR results of PDCs and the origin tissues 
Table 3 DNA fingerprinting analysis using 20 STR loci for three established GBC PDCs

\begin{tabular}{|c|c|c|c|}
\hline STR locus & JXQ-3D-902R4 & JXQ-3D-4494R & JXQ-3D-4786R \\
\hline Amelogenin & $x$ & $X, Y$ & $X, Y$ \\
\hline D5S818 & 11,12 & 11,12 & 11,13 \\
\hline D13S317 & 9,11 & 8,12 & 8,10 \\
\hline D7S820 & 8,12 & 8,11 & 11 \\
\hline D16S539 & 10,11 & 9,12 & 10,13 \\
\hline vWA & 19 & 16 & 18 \\
\hline TH01 & 9 & 7 & 7 \\
\hline TPOX & 8,12 & 9,12 & 8 \\
\hline CSF1PO & 12 & 9,12 & 11,12 \\
\hline D12S391 & 20 & 18,19 & 20 \\
\hline FGA & 25 & 23,27 & 22,24 \\
\hline D2S1338 & 19,23 & 19,23 & 19 \\
\hline D21S11 & 31,32 & 31 & $29,32.2$ \\
\hline D18S51 & 17,19 & 17 & 14 \\
\hline D8S1179 & 13 & 12,15 & 14 \\
\hline D3S1358 & 15 & 18 & 16,17 \\
\hline D6S1043 & 11 & 18,19 & 10 \\
\hline PENTAE & 12 & 12,21 & 12,17 \\
\hline D19S433 & 15.2 & 12,14 & $13,15.2$ \\
\hline PENTAD & 8,13 & 9 & 11,12 \\
\hline
\end{tabular}

GBC, gallbladder carcinoma; PDCs, patient-derived primary cell lines; STR, short tandem repeat.

showed the PDCs to have been derived from the primary GBC tissues.

After establishment, the growth characteristics of the three GBC PDCs-composed of the proliferation-related properties including cell proliferation and colony formation activities-were studied in vitro (Figure 1B,C). JXQ-3D4494R had the highest cloning efficiency among the three PDCs (Figure 1B); growth curves also showed the same tendency. At the same seeding density of 2,000 cells/well, JXQ-3D-4494R had the fastest growth rate among the cell lines (Figure $1 C$ ). The population-doubling time for JXQ3D-902R4 was $42 \mathrm{~h}$ at a density of 2,000 cells/well in 96well plates, $38 \mathrm{~h}$ at a density of 1,000 cells/well for JXQ3D-4494R, and $57 \mathrm{~h}$ for JXQ-3D-4786R at a density of 4,000 cells/well.

RNA-seq was performed to identify the gene expression of three PDCs. Each PDC was assessed three times in parallel. The typical epithelial marker genes specifically KRT7, KRT8, KRT18 and KRT19 were more highly expressed than the negative control genes (CD34 and VIM) (Table S2).

\section{Glycosylation characteristics of the three PDCs}

$\mathrm{N}$-glycans of JXQ-3D-902R4 were shown in Figure $2 A$. Without separation, 30 specific $\mathrm{N}$-glycans (linked to asparagine) of JXQ-3D-902R4 were detected. Among the $30 \mathrm{~N}$-glycans, five were of the high-mannose type, 10 were those of bi-antennary structures, and 15 were those of triantennary and tetra-antennary structures. The proposed $\mathrm{N}$-glycans and the assignment of peaks are shown in Figure $2 A$. Interestingly, about half of these $\mathrm{N}$-linked glycans contained one or two residues of sialic acid attached to terminal galactosyl, and more than half of these glycans comprised up to two residues of fucose which are linked to reducing terminal $\mathrm{N}$-acetylglucosaminide and the non-reducing end of the glycans. $17 \mathrm{~N}$-linked glycans were harvested from JXQ-3D-902R4, which equated to almost $57 \%$ of the CEA glycan moieties present core fucosylation (attached to GlcNAc) according to the study. Core fucosylation was transferred from fucose of guanosine diphosphate-fucose (GDP-Fuc) to the innermost GlcNAc with the catalysis of core fucosyltransferase. Similarly, both residues of sialic acid and residues of fucose were found in $15 \mathrm{~N}$-linked glycans of tumor antigen.

$\mathrm{N}$-glycans of JXQ-3D-4494R were shown in Figure $2 B$. Without separation, 28 specific $\mathrm{N}$-glycan of JXQ-3D4494R were detected. Among the $28 \mathrm{~N}$-glycans, five were of the high-mannose type, 11 were those of bi-antennary structures, 10 were of tri-antennary and tetra-antennary structures, and 2 bisected glycans were also determined. The proposed $\mathrm{N}$-glycans and the assignment of peaks are shown in Figure 2B. Only 12 glycans contained one or two residues of sialic acid attached to terminal galactosyl, and more than half of these glycans comprised up to two residues of fucose which are linked to reducing terminal $\mathrm{N}$-acetylglucosaminide and the non-reducing end of the glycans. Almost all the fucosylated glycans harvested from JXQ-3D-4494R presented core fucosylation (attached to GlcNAc) according to the study. Similarly, both residues of sialic acid and residues of fucose were found in $11 \mathrm{~N}$-linked glycans of tumor antigen.

$\mathrm{N}$-glcyans from JXQ-3D-4786R were shown in Figure 2C. Without separation, 29 specific $\mathrm{N}$-glycan extracted from 
A JXQ-3D-902R4

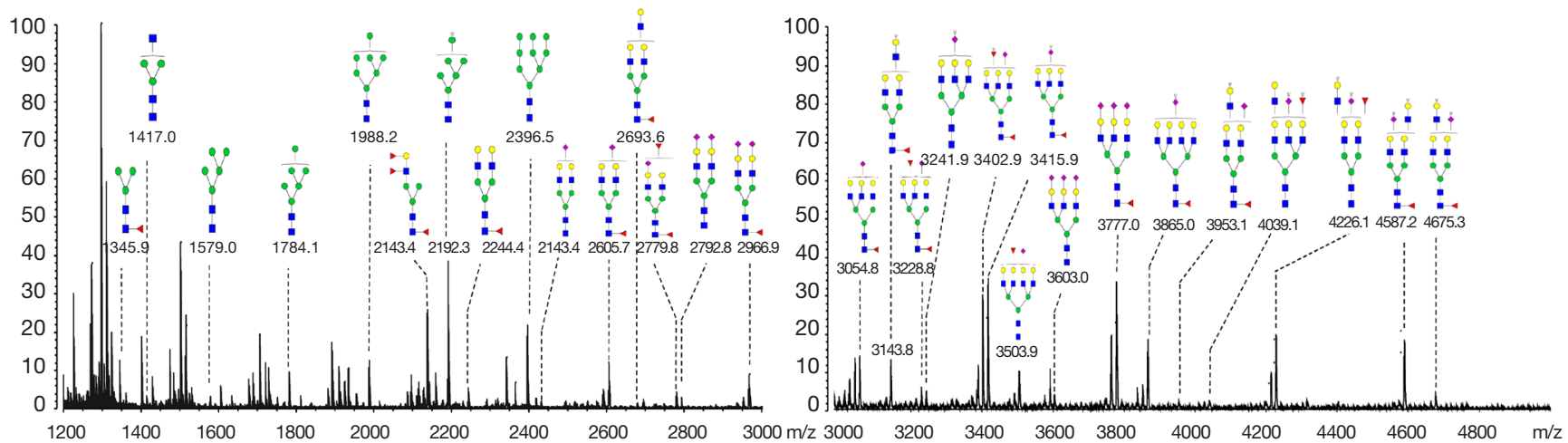

B

JXQ-3D-4494R

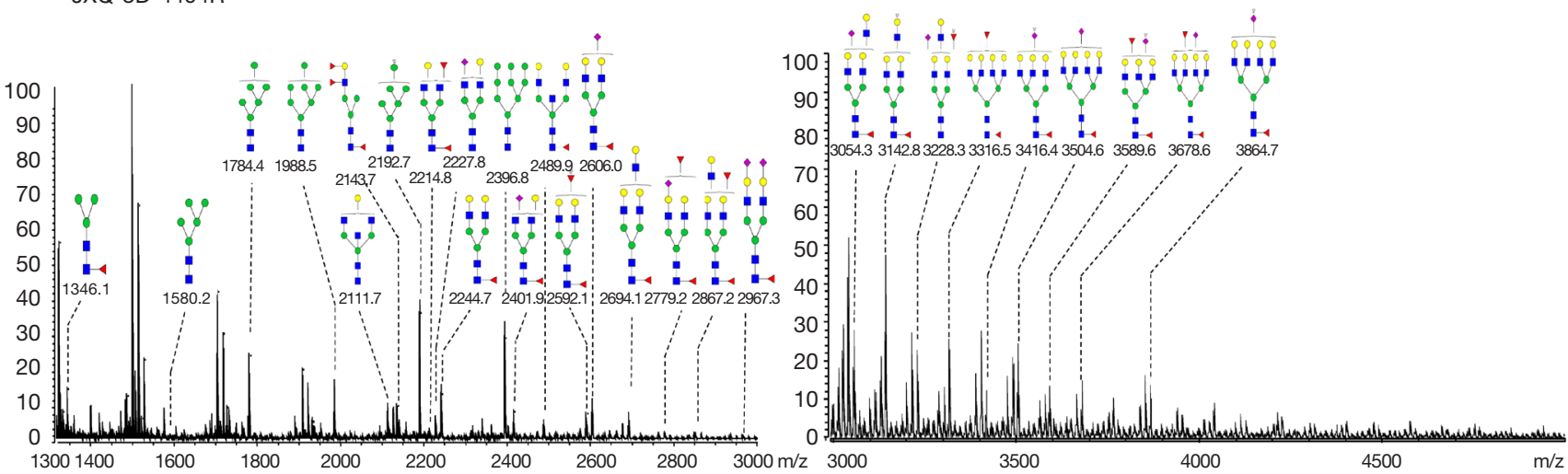

C JXQ-3D-4786R

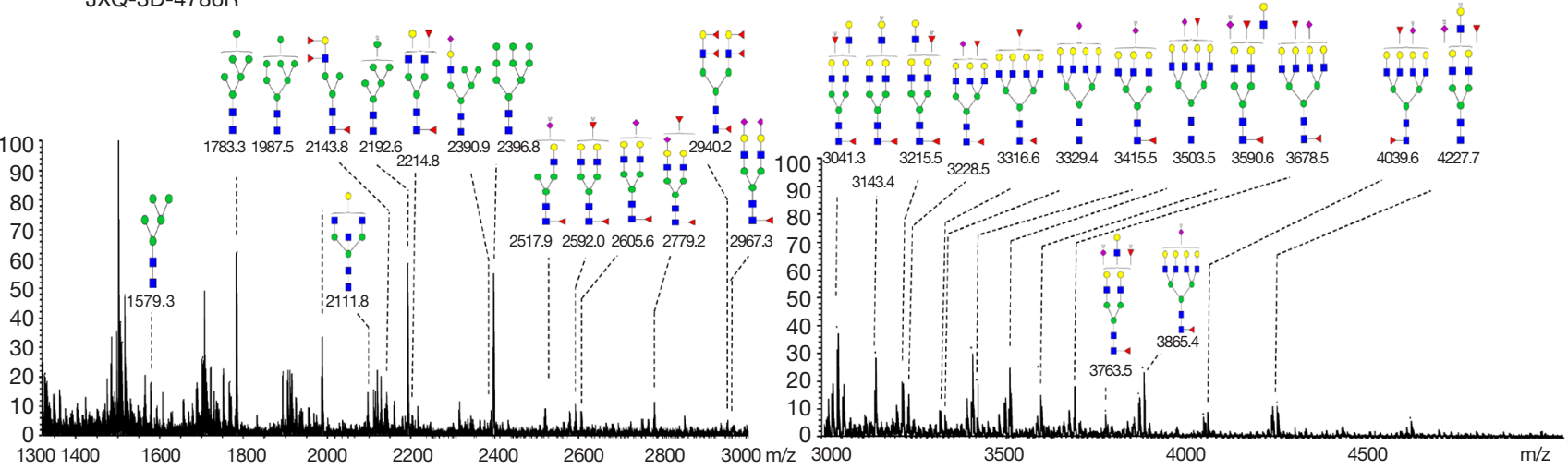

Figure 2 Glycosylation modification of three GBC PDCs. MADLI-TOF-MS was used to analyze the N-glycosylation composition of the whole protein of three GBC PDCs after methylation. N-glycans from GBC patient-derived primary cell line of JXQ-3D-902R4 (A), JXQ-3D-4494R (B), and JXQ-3D-4786R (C), respectively. N-linked glycans were slightly different in amounts for three GBC PDCs, but

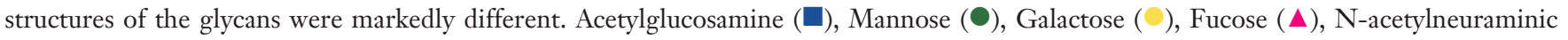
acid $(\diamond)$. GBC, gallbladder carcinoma; PDC, patient-derived primary cell line; MADLI-TOF-MS, matrix-assisted laser desorption/ ionization time of flight mass spectrometry. 
JXQ-3D-4786R were detected. Among the $29 \mathrm{~N}$-glycans, five were of the high-mannose type, 8 were those of biantennary structures, and 14 were those of tri-antennary and tetra-antennary structures. One bisected glycan and one hybrid glycan was discovered. The proposed N-glycans and the assignment of peaks are shown in Figure 2C. Fifteen glycans contained one or two residues of sialic acid attached to terminal galactosyl, and more than half of these glycans comprised up to two residues of fucose which are linked to reducing terminal $\mathrm{N}$-acetylglucosaminide and the nonreducing end of the glycans. Almost all the fucosylated glycans harvested from JXQ-3D-4786R presented core fucosylation (attached to GlcNAc) according to the study. Similarly, both residues of sialic acid and residues of fucose were found in $13 \mathrm{~N}$-linked glycans of tumor antigen.

\section{Comparisons of $\mathrm{N}$-glycans in different GBC PDCs}

$\mathrm{N}$-linked glycans were slightly different in amounts for the three PDCs, but structures of the glycans were markedly different. As shown in Table 4, ten unique N-glycans were identified from JXQ-3D-902R4, whereas four were identified for JXQ-3D-4494R, and seven were identified for JXQ-3D-4786R. For the JXQ-3D-902R4 cell line, its unique $\mathrm{N}$-glycans were mostly sialylated. For JXQ-3D4494R, unique bisecting N-glycans were detected. For JXQ-3D-4786R, unique hybrid and highly fucosylated $\mathrm{N}$-glycans were detected. As a result, these unique features in glycosylation were used to identify cell lines from different patients. In addition, $63 \%$ of the glycans were sialyted glycans in JXQ-3D-902R4, whereas there were $42 \%$ for JXQ-3D-4494R, and $51 \%$ for JXQ-3D-4786R. The ratios of core fucosylated glycans were $56 \%, 67 \%$, and $62 \%$ for JXQ-3D-902R4, JXQ-3D-4494R and JXQ-3D$4786 \mathrm{R}$, respectively.

\section{Tumorigenesis assay in vivo}

All three GBC PDCs (JXQ-3D-902R4, JXQ-3D-4494R and JXQ-3D-4786R) were transplanted subcutaneously into nude mice for tumorigenesis observations. Both transplanted xenograft models of JXQ-3D-902R4 and JXQ-3D-4494R showed kinetic growth curves (Figure 3). Transplanted xenografts of JXQ-3D-902R4 grew up to over $500 \mathrm{~mm}^{3}$ on day 44 after injection, while the ones of JXQ-3D-4494R grew up to a similar tumor volume on day 20 after injection (Figure 3). Finally, JXQ-3D-4786R did not grow to form any tumors in vivo. These results imply that JXQ-3D-4494R possesses the strongest tumorigenic ability among the three GBC PDCs. The tumorigenesis of the three PDCs also yielded the same trend similar to that of their growth activity results. Images of transplanted xenografts of JXQ-3D-902R4 and JXQ-3D-4494R were also shown (Figure S2).

\section{Diverse responses of the three PDCs to targeted drugs}

The three established GBC PDCs were used as in vitro models to study the sensitivity of targeted medicines. In the present study, seven molecular target inhibitors were used for PDCs model performance testing and evaluation: ribociclib, palbociclib, MK2206, everolimus, PI-103, lapatinib and dacomitinib. Ribociclib and palbociclib were examples of cell cycle inhibitors, whereas MK2206, everolimus and PI-103 were representative as inhibitors of the PI3K/AKT/mammalian target of rapamycin (mTOR) signaling pathway. Finally, lapatinib and dacomitinib represented examples of epidermal growth factor receptor (ErbB) inhibitors. The results demonstrated that no groups of inhibitors were effective against all three cell lines. JXQ3D-4786R was sensitive to ErbB inhibitors, and particularly insensitive to mTOR inhibitors. JXQ-3D-4494R was sensitive to most of the inhibitors, and especially sensitive to cell cycle inhibitors and ErbB inhibitors. Finally JXQ3D-902R4 was particularly sensitive to mTOR inhibitors, whereas it was insensitive to cell cycle inhibitors and ErbB inhibitors (Figure 4).

\section{Discussion}

In this study, we successfully established and characterized three GBC PDCs from three patients. Human GBC is a rare and highly fatal malignancy of the biliary tract with unclear biological mechanisms and poor prognosis. Due to the diagnosis at late stages of GBC, disappointing outcomes often result from surgical resection, chemotherapy and radiotherapy $(24,25)$. Consequently, it is important to better elucidate the mechanisms of GBC invasion and metastasis to improve the prognosis of $\mathrm{GBC}$ in the future.

The progress of cell-culture technology has facilitated the possibility of establishing a variety of human carcinoma cell lines from surgical and autopsy tissues, biopsy specimens and peritoneum effusion. Permanent cell lines established from human cancers play a major role in basic scientific studies. According to previous reports, there are already some established cancer PDCs (16). But compared to other 
Table 4 Glycosylation of three GBC PDCs

\begin{tabular}{lccc}
\hline Variable & JXQ-3D-902R4 & JXQ-3D-4494R & JXQ-3D-4786R \\
\hline Unique glycans & 10 & 4 & 7 \\
Sialylation ratio & $63 \%$ & $42 \%$ & $51 \%$ \\
Core fucosylation ratio & $56 \%$ & $67 \%$ & $62 \%$ \\
\hline
\end{tabular}

The value of the sialylation ratio is the proportion of sialylated N-glycans among all the detected glycans for each PDC. The value of core fucosylation ratio is the ratio of glycans with a fucose residue in the reducing terminal among all the detected glycans for each PDC. GBC, gallbladder carcinoma; PDCs, patient-derived primary cell lines.
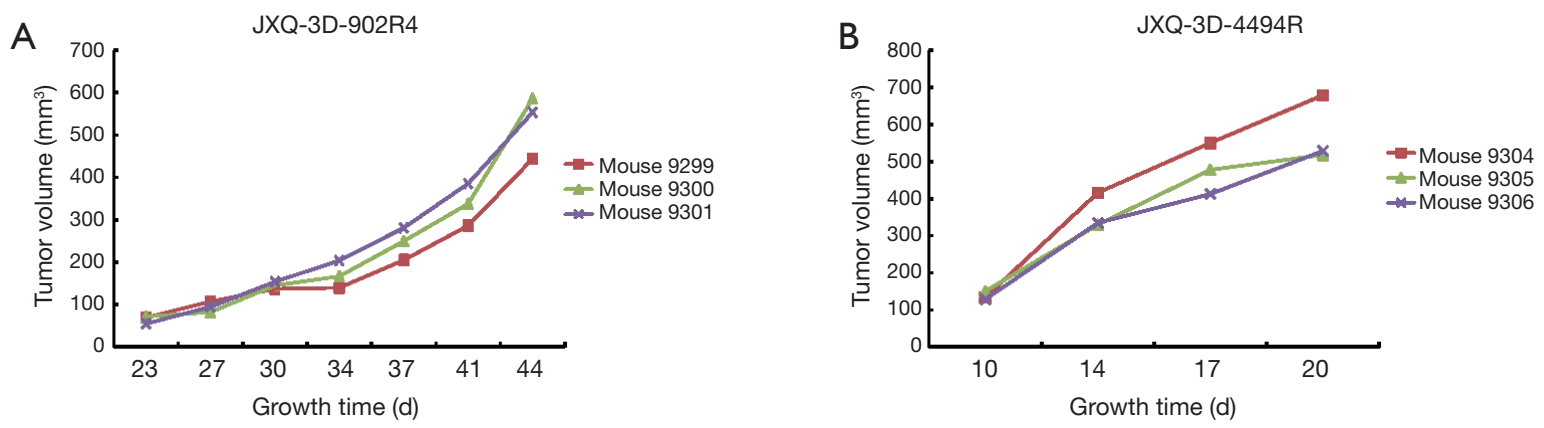

Figure 3 Tumorigenicity of three GBC PDCs in naked mice. JXQ-3D-902R4, JXQ-3D-4494R and JXQ-3D-4786R were subcutaneously transplanted into nude mice for tumorigenesis observation. Transplanted xenografts volume which grew up to over $500 \mathrm{~mm}^{3}$ in 44 days and kinetic growth curve is shown. 9299, 9300 and 9301 are the mouse number labels (A). The transplanted xenografts of JXQ-3D-4494R grew up to a similar tumor volume in 20 days. 9304, 9305 and 9306 are also the mouse number labels (B). However, JXQ-3D-4786R did not grow to form any tumors in vivo due to the poor growth rate. GBC, gallbladder carcinoma; PDC, patient-derived primary cell line.

types of cancer, there are relatively few available GBC PDCs (26). Gallbladder adenocarcinomas are the major type of GBC, comprising approximately $80-97 \%$ of GBCs (27). We successfully established three PDCs, which were all derived from patients with gallbladder adenocarcinomas. Our present study comprehensively described the characteristics of the three PDCs in vitro, including the glycosylation modifications of total proteins. The three primary PDCs showed morphological heterogeneity with different cell shapes (Figure 1A). The growth rates were also different. JXQ-3D-4786R grew at the lowest speed, whereas JXQ-3D-4494R grew at the highest speed (Figure $1 B, C)$. We also detected the growth activity of the three cell lines in vivo. Our tumorigenesis assay in vivo showed the same trend that we found in vitro. That is, JXQ-3D4494R and JXQ-3D-902R4 grew quickly to successfully form tumors subcutaneously in naked mice, whereasdue to it having the slowest growth rate-JXQ-3D-4786R did not form any tumors subcutaneously in naked mice (Figure 2). These results demonstrate the heterogeneity of growth ability within the three PDCs. In this study, we first described the characteristics of glycosylation modification of the PDCs. From these results, we found the unique glycans, sialylation ratio and core fucosylation ratio of each PDC (Table 4). The glycosylation-modification features reveal the uniqueness of each PDC, and the glycosylation of the whole proteins also provide novel clues for future functional studies. Glycosylation reflects the coordinated effort of a complex array of enzymes, organelles, and other factors that are needed to successfully generate carbohydrateassociated post-translational modifications. Due to the significant role of glycans in the molecular composition of a glycoprotein, alterations in glycosylation can significantly impact overall glycoprotein charge and conformation and therefore readily alter its biological activity. Furthermore, branches are biosynthesized with the help of a particular glycosyltransferase, and relatively simple changes in one glycosyltransferase can impact the biology of many different proteins in meaningful ways, thus enabling rather subtle genetic changes to induce highly pleiotropic effects on cancer cell survival and overall progression $(28,29)$. In this study, we explored the glycosylation characteristics 


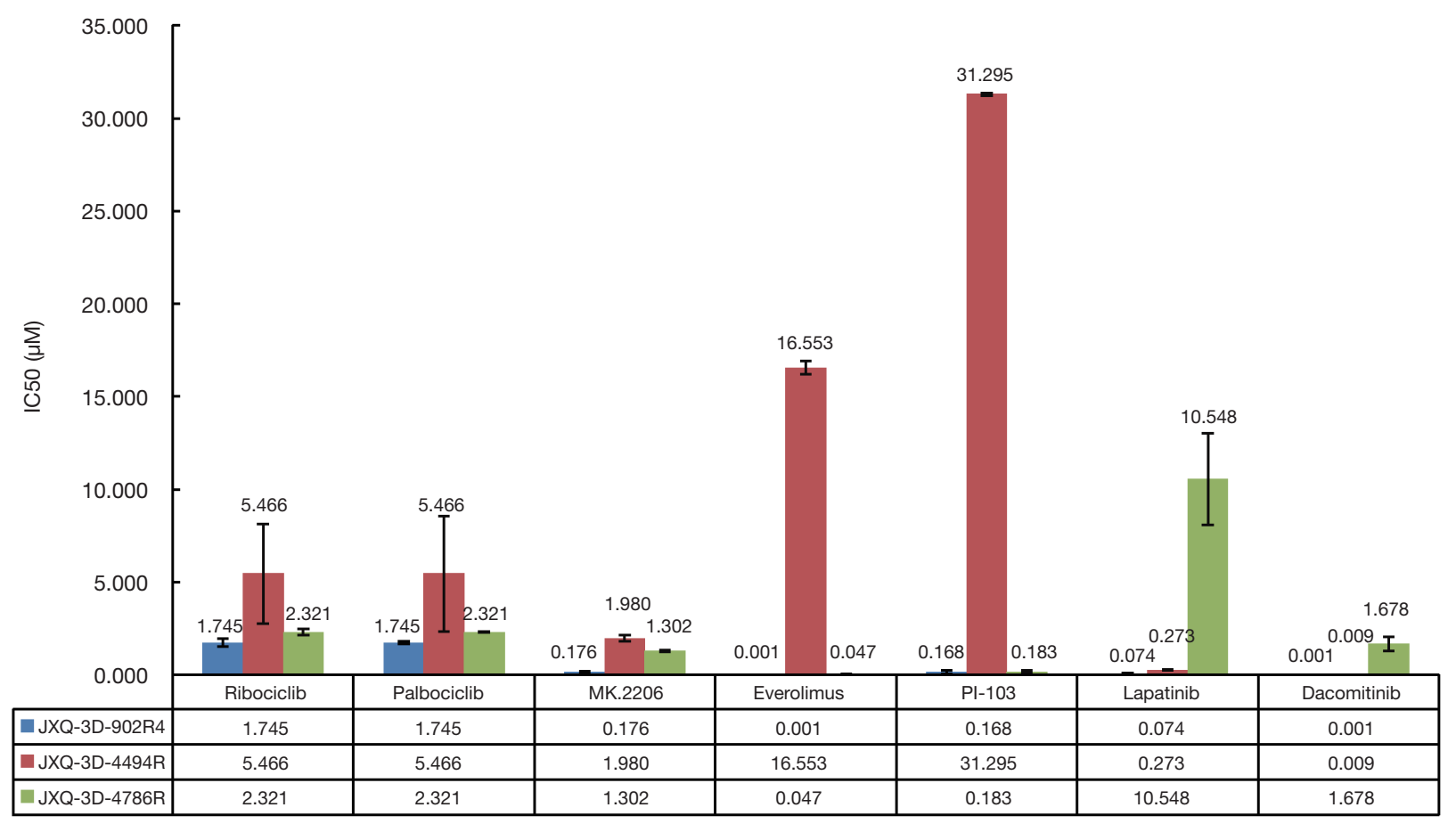

Figure 4 Sensitivities of the three PDCs to targeted drugs for gallbladder carcinoma. Fifty percent growth inhibitory concentrations (IC50s) of seven-drugs—ribociclib, palbociclib, MK2206, everolimus, PI-103, lapatinib and dacomitinib—on JXQ-3D-902R4, JXQ-3D-4494R and JXQ-3D-4786R are indicated. Ribociclib and palbociclib were cell cycle inhibitors; MK2206, everolimus and PI-103 were inhibitors of the $\mathrm{PI} 3 \mathrm{~K} / \mathrm{AKT} / \mathrm{mTOR}$ signaling pathway; lapatinib and dacomitinib were ErbB inhibitors. This experiment was biologically replicated three times. The original cell density of the three PDCs: JXQ-3D-4494R, 1,000 cells/well; JXQ-3D-4786R, 4,000 cells/well; JXQ-3D-902R4, 2,000 cells/well. Cell viability was measured after $144 \mathrm{~h}$ incubation. Error bars represent mean +/- SD from three replicates. The numbers in the table are the IC50s $(\mu \mathrm{M})$ of the seven drugs. GBC, gallbladder carcinoma; PDC, patient-derived primary cell line; IC50, Fifty percent growth inhibitory concentrations; ErbB, epidermal growth factor receptor; mTOR, mammalian target of rapamycin.

of the three PDCs. The limitations of the assay were that we described only the glycan features. Due to the absence of normal gallbladder cells as controls, it was difficult to further investigate the biological and clinical significance of the glycosylation.

Development of medicines for cancer therapy has been hampered by a lack of preclinical models. An appropriate preclinical tool could reliably predict the efficacy of novel drugs in cancer therapy. Patient-derived tumor xenografts (PDXs) or cells (PDCs) are regarded as appropriate tools and models for basic clinical research and have been increasingly employed over the last few decades (30-33). In the present study, we presented the efficacy outcome of seven chemo-drugs evaluated by our three successfully established PDCs. The seven compounds belong to the following three groups: cell cycle inhibitors, PI3K/AKT/ mTOR signaling-pathway inhibitors, and ErbB inhibitors.
The in vitro anti-proliferation assay in all of the PDCs indicated the different sensitivities of the three PDCs to the seven-most used chemo-drugs. None of the drugs were effective against all three PDCs (Figure 4). After comprehensive evaluation and comparison of the IC50 values of the seven drugs against the three PDCs, we recommend that MK2206 and Dacomitinib should be used for further research because both drugs exhibited the highest relative inhibitory effect against all of the three PDCs. Considering the complexity of the development of chemoresistance, PDCs may be qualified as preclinical research models to assess the efficacy of cancer medicines.

In conclusion, we successfully established and characterized three PDCs derived from GBC patients. The three PDCs presented herein enrich the pool of primary cell lines of GBC. In this study, we described their morphology and growth characteristics, and we first demonstrated their 
glycosylation-modification features. Finally, we used these well-characterized PDCs to evaluate the efficacy of seven targeted drugs. These results suggest that these PDCs have the potential to be valuable tools for preclinical studies of targeted therapies for human GBC.

\section{Acknowledgments}

Funding: This study was supported by Special Fund on the Application and Transformation of Precision Medicine in Second Military Medical University (No. 2017JZ11).

\section{Footnote}

Conflicts of Interest: All authors have completed the ICMJE uniform disclosure form (available at http://dx.doi. org/10.21037/tcr.2020.02.04). The authors have no conflicts of interest to declare.

Ethical Statement: The authors are accountable for all aspects of the work in ensuring that questions related to the accuracy or integrity of any part of the work are appropriately investigated and resolved. The study was conducted in accordance with the Declaration of Helsinki (as revised in 2013). Gallbladder carcinoma tissues of patients were collected with ethics approval obtained from the Eastern Hepatobiliary Surgery Hospital (No. EHBHKY2015-02-010), and informed consent was obtained from the patients for PDC culture and further research.

Open Access Statement: This is an Open Access article distributed in accordance with the Creative Commons Attribution-NonCommercial-NoDerivs 4.0 International License (CC BY-NC-ND 4.0), which permits the noncommercial replication and distribution of the article with the strict proviso that no changes or edits are made and the original work is properly cited (including links to both the formal publication through the relevant DOI and the license). See: https://creativecommons.org/licenses/by-nc-nd/4.0/.

\section{References}

1. Hundal R, Shaffer EA. Gallbladder cancer: epidemiology and outcome. Clin Epidemiol 2014;6:99-109.

2. Lazcano-Ponce EC, Miquel JF, Munoz N, et al. Epidemiology and molecular pathology of gallbladder cancer. CA Cancer J Clin 2001;51:349-64.
3. Shen HX, Song HW, Xu XJ, et al. Clinical epidemiological survey of gallbladder carcinoma in northwestern China, 2009-2013: 2379 cases in 17 centers. Chronic Dis Transl Med 2017;3:60-6.

4. de Groen PC, Gores GJ, LaRusso NF, et al. Biliary tract cancers. N Engl J Med 1999;341:1368-78.

5. Hueman MT, Vollmer CM Jr, Pawlik TM. Evolving treatment strategies for gallbladder cancer. Ann Surg Oncol 2009;16:2101-15.

6. Miyakawa S, Ishihara S, Horiguchi A, et al. Biliary tract cancer treatment: 5,584 results from the Biliary Tract Cancer Statistics Registry from 1998 to 2004 in Japan. J Hepatobiliary Pancreat Surg 2009;16:1-7.

7. Hong EK, Kim KK, Lee JN, et al. Surgical outcome and prognostic factors in patients with gallbladder carcinoma. Korean J Hepatobiliary Pancreat Surg 2014;18:129-37.

8. Zhu AX, Hong TS, Hezel AF, et al. Current management of gallbladder carcinoma. Oncologist 2010;15:168-81.

9. Sekine S, Shimada Y, Nagata T, et al. Establishment and characterization of a new human gallbladder carcinoma cell line. Anticancer Res 2012;32:3211-8.

10. Kodack DP, Farago AF, Dastur A, et al. Primary PatientDerived Cancer Cells and Their Potential for Personalized Cancer Patient Care. Cell Rep 2017;21:3298-309.

11. Pan C, Kumar C, Bohl S, et al. Comparative proteomic phenotyping of cell lines and primary cells to assess preservation of cell type-specific functions. Mol Cell Proteomics 2009;8:443-50.

12. Alge CS, Hauck SM, Priglinger SG, et al. Differential protein profiling of primary versus immortalized human RPE cells identifies expression patterns associated with cytoskeletal remodeling and cell survival. J Proteome Res 2006;5:862-78.

13. Bissig-Choisat B, Kettlun-Leyton C, Legras XD, et al. Novel patient-derived xenograft and cell line models for therapeutic testing of pediatric liver cancer. J Hepatol 2016;65:325-33.

14. Chapuy B, Cheng H, Watahiki A, et al. Diffuse large B-cell lymphoma patient-derived xenograft models capture the molecular and biological heterogeneity of the disease. Blood 2016;127:2203-13.

15. Nicolle D, Fabre M, Simon-Coma M, et al. Patientderived mouse xenografts from pediatric liver cancer predict tumor recurrence and advise clinical management. Hepatology 2016;64:1121-35.

16. Liu ZY, Xu GL, Tao HH, et al. Establishment and characterization of a novel highly aggressive gallbladder cancer cell line, TJ-GBC2. Cancer Cell Int 2017;17:20. 
17. Li QK, Chen L, Ao MH, et al. Serum fucosylated prostatespecific antigen (PSA) improves the differentiation of aggressive from non-aggressive prostate cancers. Theranostics 2015;5:267-76.

18. Zhao Q, Zhan T, Deng Z, et al. Glycan analysis of colorectal cancer samples reveals stage-dependent changes in CEA glycosylation patterns. Clin Proteomics 2018;15:9.

19. Yang G, Tan Z, Lu W, et al. Quantitative glycome analysis of $\mathrm{N}$-glycan patterns in bladder cancer vs normal bladder cells using an integrated strategy. J Proteome Res 2015;14:639-53.

20. Sethi MK, Thaysen-Andersen M, Smith JT, et al. Comparative $\mathrm{N}$-glycan profiling of colorectal cancer cell lines reveals unique bisecting GlcNAc and alpha2,3-linked sialic acid determinants are associated with membrane proteins of the more metastatic/aggressive cell lines. J Proteome Res 2014;13:277-88.

21. Feng F, Cheng Q, Yang L, et al. Guidance to rational use of pharmaceuticals in gallbladder sarcomatoid carcinoma using patient-derived cancer cells and whole exome sequencing. Oncotarget 2017;8:5349-60.

22. Kohli M, Ho Y, Hillman DW, et al. Androgen Receptor Variant AR-V9 Is Coexpressed with AR-V7 in Prostate Cancer Metastases and Predicts Abiraterone Resistance. Clin Cancer Res 2017;23:4704-15.

23. Huang C, Liu Y, Wu H, et al. Characterization of IgG glycosylation in rheumatoid arthritis patients by MALDITOF-MS(n) and capillary electrophoresis. Anal Bioanal Chem 2017;409:3731-9.

24. Mastoraki A, Papanikolaou IS, Konstandiadou I, et al.

Cite this article as: Feng F, Huang C, Xiao M, Wang H, Gao Q, Chen Z, Xu X, Zhou J, Li F, Li Y, Zhang D, Chang Y, Jiang X. Establishment and characterization of patient-derived primary cell lines as preclinical models for gallbladder carcinoma. Transl Cancer Res 2020;9(3):1698-1710. doi: 10.21037/tcr.2020.02.04
Facing the challenge of treating gallbladder carcinoma. Review of the literature. Hepatogastroenterology 2010;57:215-9.

25. Shukla PJ, Barreto SG. Gallbladder cancer: we need to do better! Ann Surg Oncol 2009;16:2084-5.

26. Khan SA, Thomas HC, Davidson BR, et al. Cholangiocarcinoma. Lancet 2005;366:1303-14.

27. Goetze TO. Gallbladder carcinoma: Prognostic factors and therapeutic options. World J Gastroenterol 2015;21:12211-7.

28. Fuster MM, Esko JD. The sweet and sour of cancer: glycans as novel therapeutic targets. Nat Rev Cancer 2005;5:526-42.

29. Cummings RD, Pierce JM. The challenge and promise of glycomics. Chem Biol 2014;21:1-15.

30. Pandrangi SL, Raju Bagadi SA, Sinha NK, et al. Establishment and characterization of two primary breast cancer cell lines from young Indian breast cancer patients: mutation analysis. Cancer Cell Int 2014;14:14.

31. Tentler JJ, Tan AC, Weekes CD, et al. Patient-derived tumour xenografts as models for oncology drug development. Nat Rev Clin Oncol 2012;9:338-50.

32. Siolas D, Hannon GJ. Patient-derived tumor xenografts: transforming clinical samples into mouse models. Cancer Res 2013;73:5315-9.

33. Julien S, Merino-Trigo A, Lacroix L, et al. Characterization of a large panel of patient-derived tumor xenografts representing the clinical heterogeneity of human colorectal cancer. Clin Cancer Res 2012;18:5314-28. 


\begin{tabular}{|l|l|l|l|l|l|l|l|l|l|l|l|l|}
\hline & 1 & 2 & 3 & 4 & 5 & 6 & 7 & 8 & 9 & 10 & 11 & 12 \\
\hline A & & & & & & & & & & & & \\
\hline B & & & & & & & & & & & & \\
\hline C & JXQ-3D-902R4 & 4000 & 4000 & 4000 & 2000 & 2000 & 2000 & 1000 & 1000 & 1000 & NcC & \\
\hline D & JXQ-3D-4494R & 4000 & 4000 & 4000 & 2000 & 2000 & 2000 & 1000 & 1000 & 1000 & NcC & \\
\hline E & JXQ-3D-4786R & 4000 & 4000 & 4000 & 2000 & 2000 & 2000 & 1000 & 1000 & 1000 & NcC & \\
\hline F & & & & & & & & & & & & \\
\hline G & & & & & & & & & & & & \\
\hline H & & & & & & & & & & & & \\
\hline
\end{tabular}

Figure S1 The experimental design of cell proliferation assay. The numbers in the image represent the cell density used in the experiment, unit: cells/well. NcC, no cell control.

JXQ-3D-902R4

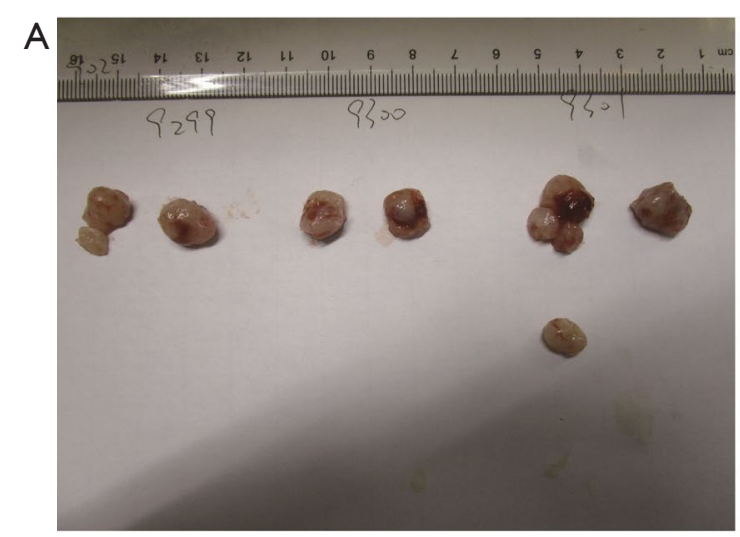

JXQ-3D-4494R

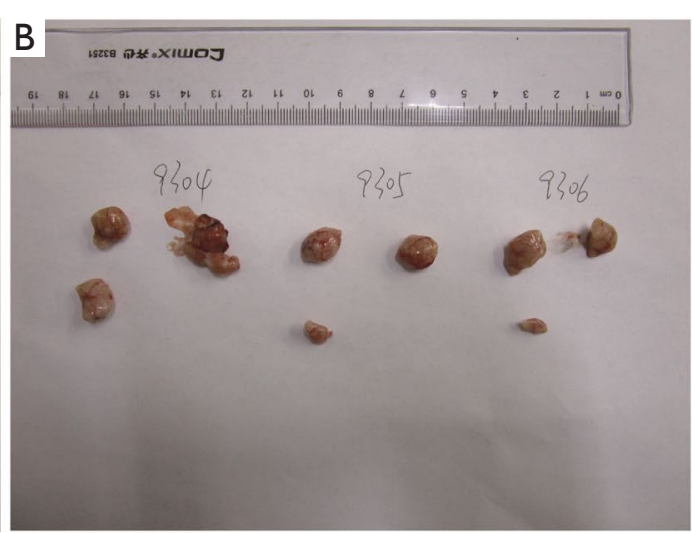

Figure S2 Images of transplanted xenografts of JXQ-3D-902R4 (A) and JXQ-3D-4494R (B). 
Table S1 STR loci analysis for primary tissues of three established GBC PDCs

\begin{tabular}{|c|c|c|c|}
\hline STR locus & JXQ-3D-902R4 & JXQ-3D-4494R & JXQ-3D-4786R \\
\hline Amelogenin & $x$ & $X, Y$ & $X, Y$ \\
\hline D5S818 & 11,12 & 11,12 & 11,13 \\
\hline D13S317 & 9,11 & 8,12 & 8,10 \\
\hline D7S820 & 8,12 & 8,11 & 8,11 \\
\hline D16S539 & 10,11 & 9,12 & 10,13 \\
\hline vWA & 16,19 & 16 & 17,18 \\
\hline TH01 & 9 & 7 & 7 \\
\hline TPOX & 8,12 & 9,12 & 8 \\
\hline CSF1PO & 12 & 9,12 & 11 \\
\hline D12S391 & 20,22 & 18,19 & 19,20 \\
\hline FGA & 22,25 & 23,27 & 22 \\
\hline D2S1338 & 19,23 & 19,23 & 19,24 \\
\hline D21S11 & 31,32 & $31,33.2$ & $29,32.2$ \\
\hline D18S51 & 17,19 & 17 & 14,23 \\
\hline D8S1179 & 13,14 & 12,15 & 14 \\
\hline D3S1358 & 15 & 14,18 & 16,17 \\
\hline D6S1043 & 11 & 18 & 10,14 \\
\hline PENTAE & 12,13 & 12,21 & 12,17 \\
\hline D19S433 & 15.2 & 12,14 & $13,15.2$ \\
\hline PENTAD & 8,13 & 9 & 11,12 \\
\hline
\end{tabular}

GBC, gallbladder carcinoma; PDC, patient-derived primary cell line; STR, short tandem repeat.

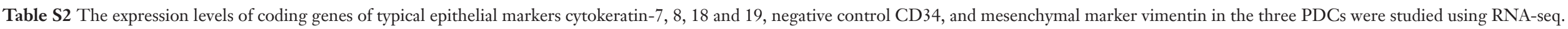
Each PDC was assessed three times in parallel

\begin{tabular}{|c|c|c|c|c|c|c|c|c|c|}
\hline Gene & JXQ-3D-4494R-1 & JXQ-3D-4494R-2 & JXQ-3D-4494R-3 & JXQ-3D-4786R-1 & JXQ-3D-4786R-2 & JXQ-3D-4786R-3 & JXQ-3D-902R4-1 & JXQ-3D-902R4-2 & JXQ-3D-902R4-3 \\
\hline KRT7 & 700.28 & 669.813 & 720.693 & $1,107.91$ & $1,125.34$ & $1,134.48$ & $1,179.14$ & $1,109.07$ & $1,243.15$ \\
\hline KRT8 & 380.893 & 383.784 & 395.832 & 338.43 & 336.079 & 344.675 & 812.417 & 760.385 & 842.105 \\
\hline KRT18 & 307.122 & 297.565 & 304.337 & 141.444 & 146.995 & 146.672 & 949.329 & 910.485 & 962.935 \\
\hline KRT19 & 801.223 & 798.285 & 837.328 & $2,477.54$ & $2,406.53$ & $2,478.08$ & 481.031 & 443.964 & 493.705 \\
\hline$C D 34$ & 0.150532 & 0.124826 & 0.167466 & 0 & 0.0405161 & 0.0428595 & 0.0168717 & 0.0168319 & 0.0383703 \\
\hline VIM & 3.9862 & 3.98274 & 4.03764 & 0.144452 & 0.152615 & 0.125598 & 14.813 & 15.12 & 13.5235 \\
\hline
\end{tabular}

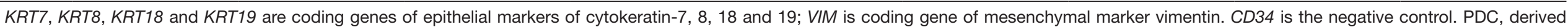
primary cell. 